\title{
Colour flow imaging in the diagnosis of multiple ventricular septal defects
}

\author{
GEORGE R SUTHERLAND, JOHN H SMYLLIE, BRUCE C OGILVIE, \\ BARRY R KEETON
}

From the Department of Paediatric Cardiology, Wessex Cardiothoracic Unit, Southampton General Hospital, Southampton

SUMMARY Thirty one patients with multiple ventricular septal defects were studied by cross sectional echocardiography, conventional pulsed and continuous wave Doppler, colour flow imaging, and left ventriculography to determine the relative diagnostic benefits and pitfalls of each technique. The patients studied had a wide range of congenital heart defects with 19 patients having isolated multiple ventricular septal defects, three with associated tetralogy of Fallot, five with double outlet right ventricle, three with complete transposition and ventricular septal defect, and one with a complete atrioventricular septal defect. In 23 patients the defects were inspected at operation. Cross sectional imaging with integrated pulsed and continuous wave Doppler correctly identified multiple defects in only $12(39 \%)$ patients. In contrast, colour flow imaging was accurate in $24(77 \%)$ patients and left ventriculography in $20(65 \%)$ patients. When patients were subdivided on the basis of relative peak systolic ventricular pressures into restrictive defects (18 patients) and non-restrictive defects (13 patients) the diagnostic value of colour flow imaging was different for each group. Colour flow mapping correctly identified multiple ventricular septal defects in $16 / 18(89 \%)$ patients with restrictive defects but only $8 / 13(62 \%)$ with non-restrictive defects. The comparative diagnostic accuracy of left ventriculography was $15 / 18(83 \%)$ in the restrictive group and 5/13 (38\%) in the non-restrictive group.

Colour flow imaging was the single investigative technique with the greatest diagnostic accuracy in the diagnosis of multiple ventricular septal defects. It failed to be consistently accurate in defined subgroups with non-restrictive defects as did left ventriculography. The greatest overall diagnostic accuracy in this series was obtained when both colour flow imaging and ventriculography techniques were used in combination in a complementary fashion.

Multiple ventricular septal defects are not uncommon in congenital heart disease. The reported frequency in patients with multiple ventricular septal defects as an isolated lesion ranges from $4 \%$ to $18 \%{ }^{1-3}$ Multiple ventricular septal defects are more common in patients with double outlet right ventricle, ${ }^{4}$ complete atrioventricular septal defects, ${ }^{56}$ tetralogy of Fallot, ${ }^{5}$ and complete transposition with ventricular septal defect. ${ }^{5}$

It is important to make a precise preoperative diagnosis of multiple ventricular septal defects

Requests for reprints to Dr George R Sutherland, Thoraxcentre, Dijktzigt Academic Hospital, 3000 DR Rotterdam, The Netherlands.

Accepted for publication 24 January 1989 because they are associated with a higher surgical mortality ${ }^{2}$ and a higher rate of palliative pulmonary artery banding rather than primary repair; failure to make an accurate preoperative diagnosis leads to a high reoperation rate. ${ }^{7}$ Traditionally the diagnosis of multiple ventricular septal defects has relied on left ventriculography. ${ }^{8}$ Despite recent improvements in this technique such as the introduction of biplane equipment for axially angled projections and the decreased toxicity of contrast agents (allowing multiple injections), the diagnostic accuracy of left ventriculography at best only approaches $86 \%$.

Ultrasound studies have shown that cross sectional echocardiography can visualise most moderate to large defects especially when they are sited in the perimembranous and the smooth muscular inlet septa. ${ }^{9}$ However, small defects both single and mul- 
tiple within the trabecular and muscular outlet septa have proved difficult to visualise by cross sectional imaging alone. ${ }^{9}$ The addition of pulsed Doppler to ultrasound systems has improved the diagnosis of small defects by identifying the diagnostic disturbances of transseptal and right ventricular flow associated with these defects. ${ }^{10}$ Although it has been suggested that multiple defects can be detected by a right ventricular and septal scanning technique with pulsed Doppler, ${ }^{10}$ others have shown that such an integrated technique of cross sectional imaging and pulsed Doppler scanning can be inaccurate in the diagnosis of multiple ventricular septal defects. ${ }^{11}$ Both pulsed and continuous wave Doppler techniques are inadequate where non-restrictive ventricular septal defects are present. These result in low transseptal flow velocities and a laminary flow across the defect, ${ }^{10}$ in contrast with the high velocity turbulent flow associated with restrictive defects.

Colour flow imaging was a useful technique for the diagnosis of single restrictive ventricular septal defects because it allowed visualisation of their characteristically turbulent transseptal and right ventricular flow patterns. ${ }^{12}$ Previous work using colour flow imaging in the diagnosis of multiple ventricular septal defects (mainly confined to restrictive defects) suggested both a high sensitivity and specificity for their detection. ${ }^{11}$ However, no direct comparison between the predictive value of colour flow imaging and left ventriculography could be made in the reported series, because only patients with multiple ventricular septal defects previously identified by left ventriculography were studied. Also no comparison was made between the diagnostic role of colour flow imaging in groups with restrictive and non-restrictive defects.

The aims of the present study were to evaluate the usefulness of colour flow imaging in the diagnosis of all types of multiple ventricular septal defects, to compare this prospectively with the diagnostic accuracy of left ventriculography, and to define any inherent limitations in the technique.

\section{Patients and methods}

\section{STUDY PATIENTS AND TYPE OF VENTRICULAR}

SEPTAL DEFECT

Between June 1986 and October 1987, 31 infants and children (aged 2 days to 11 years, mean $2 \cdot 11$ years) with multiple ventricular septal defects diagnosed at the Wessex Paediatric Cardiac Unit were investigated by angiography, cross sectional echocardiography, and colour flow imaging. Nineteen patients had isolated multiple ventricular septal defects, 11 of whom had a combination of perimembranous and muscular defects, six had multiple
Sutherland, Smyllie, Ogilvie, Keeton $\stackrel{\frac{\text { D }}{\square}}{\stackrel{T}{\mathbb{T}}}$ discrete muscular defects, and two had "Swiss cheese" defects. Of the 12 with ventricular septal $\stackrel{\complement}{=}$ defects occurring as part of a complex congenital $\overrightarrow{\vec{s}}$ lesion included in the series, three patients with tetralogy of Fallot and three patients with double $\frac{C}{O}$ outlet right ventricle had a combination of a peri- $\overline{\bar{m}}$ membranous outlet plus muscular ventricular septal $\mathbb{D}$ defects. Two further patients with double outlet right ventricle had non-committed multiple mus- ${ }^{\circ}$ cular defects. Three patients with complete trans- $\vec{\circ}$ position and multiple ventricular septal defects had a $\overrightarrow{\vec{H}}$ combination of a single perimembranous plus a $\stackrel{\omega}{\omega}$ muscular defect and the patient with a complete atrioventricular septal defect had an associated mus- of cular defect (table 1).

Patients were entered into the study only if an unequivocal diagnosis of multiple ventricular septal $\omega$ defects was first made by: (a) an unequivocally diagnostic left ventriculogram (16 patients), (b) cross $\vec{c}$ sectional imaging plus positive colour flow imaging (10 patients), and (c) surgical inspection (five patients had multiple ventricular septal defects identified for the first time at operation). All patients then underwent any of the other preoperative investigations not already undertaken, so that every patient in the study had available for review cross sectional echocardiography with conventional Doppler studies, colour flow imaging studies, and left ventriculography. The five patients whose diagnosis of multiple ventricular $\overrightarrow{\vec{O}}$ septal defects was first made at operation all had 3 preoperative left ventriculograms, cross sectional imaging, and conventional Doppler and colour flow studies, which were then reviewed. To date a total of 23 patients have had surgical closure of their ven-

Table 1 Morphological diagnosis versus type of ventricular septal defect

\begin{tabular}{|c|c|c|c|c|c|}
\hline \multirow{2}{*}{$\begin{array}{l}\text { Morphological } \\
\text { diagnosis }\end{array}$} & \multicolumn{2}{|l|}{ Restrictive } & \multicolumn{2}{|l|}{ Non-restrictive } & \multirow[b]{2}{*}{ Total } \\
\hline & Defect type & $N_{0}$ & Defect type & No & \\
\hline $\begin{array}{l}\text { Isolated VSDs } \\
\text { (no other lesion) }\end{array}$ & $\begin{array}{l}\text { PMO + musc } \\
\text { Mult musc }\end{array}$ & $\begin{array}{r}11 \\
5\end{array}$ & $\begin{array}{l}\text { Mult musc } \\
\text { "Swiss cheese" }\end{array}$ & $\begin{array}{l}1 \\
2\end{array}$ & 19 \\
\hline $\begin{array}{l}\text { Tetralogy of } \\
\text { Fallot } \\
\text { DORV }\end{array}$ & & $\begin{array}{l}0 \\
0\end{array}$ & $\begin{array}{l}\text { PMO + musc } \\
\text { PMO + musc } \\
\text { Mult musc }\end{array}$ & $\begin{array}{l}3 \\
3 \\
2\end{array}$ & $\begin{array}{l}3 \\
5\end{array}$ \\
\hline $\begin{array}{l}\text { TGA/VSD } \\
\text { CAVSD } \\
\text { Total }\end{array}$ & $\begin{array}{l}\text { PMO + musc } \\
\text { PMI + musc }\end{array}$ & $\begin{array}{r}1 \\
1 \\
0 \\
18\end{array}$ & $\begin{array}{l}\text { PMO + musc } \\
\text { PMI + musc }\end{array}$ & $\begin{array}{r}1 \\
1 \\
13\end{array}$ & $\begin{array}{r}3 \\
1 \\
31\end{array}$ \\
\hline
\end{tabular}

VSD, ventricular septal defect; PMO + musc, perimembranous outlet plus muscular defects; Mult musc, multiple muscular defects; PMI + musc, perimembranous inlet plus muscular defects; DORV, double outlet right ventricle; TGA/VSD, $\sigma$ complete transposition of the great arteries with associated $<$ ventricular septal defect; CAVSD, complete atrioventricular septal defect. 
tricular septal defects with concomitant inspection of the defect, and a further five patients have had pulmonary artery banding as an initial procedure.

\section{METHODS}

Echocardiographic studies were performed in the standard manner as described in our work on the imaging of isolated ventricular septal defects. ${ }^{9}$ Cross sectional images were obtained with an ATL Ultramark IV mechanical sector scanner with 5.0 and 7.5 $\mathrm{MHz}$ transducers, to achieve high resolution images. Conventional Doppler (both pulsed and continuous wave) and colour flow imaging studies were then obtained by a Toshiba SSH 65 A with 2.5 , 3.7 , and $5.0 \mathrm{MHz}$ phased array transducers. Colour flow studies were performed in both the power and the variance modes with appropriate gain and filter settings. The diagnosis by colour flow imaging of a single ventricular septal defect was considered definitive when colour flow (either turbulent or laminar) was shown traversing the interventricular septum. Where no transseptal flow was visualised the presence of a restrictive defect was inferred if a high velocity or turbulent jet was identified exiting from the right ventricular aspect of the septum into the right ventricular cavity. In restrictive defects the transseptal and right ventricular flow disturbance when visualised was invariably high velocity and turbulent, so a mosaic pattern was seen on the variance colour flow map (fig 1). In non-restrictive defects transseptal systolic flow was invariably of relatively low velocity and laminar (fig 2 ) with no turbulence being colour encoded. Transseptal flow within the multiple defects was therefore frequently best depicted by colour flow imaging in the power mode. Colour $M$ mode studies, in the velocity mode, were also performed in non-restrictive defects because in theory systolic flow acceleration could occur either within the $\operatorname{defect}(\mathrm{s})$ or at the right ventricle exit point without turbulence being present (fig 3). The inherently higher sampling rate of colour $M$ mode should make this method more sensitive

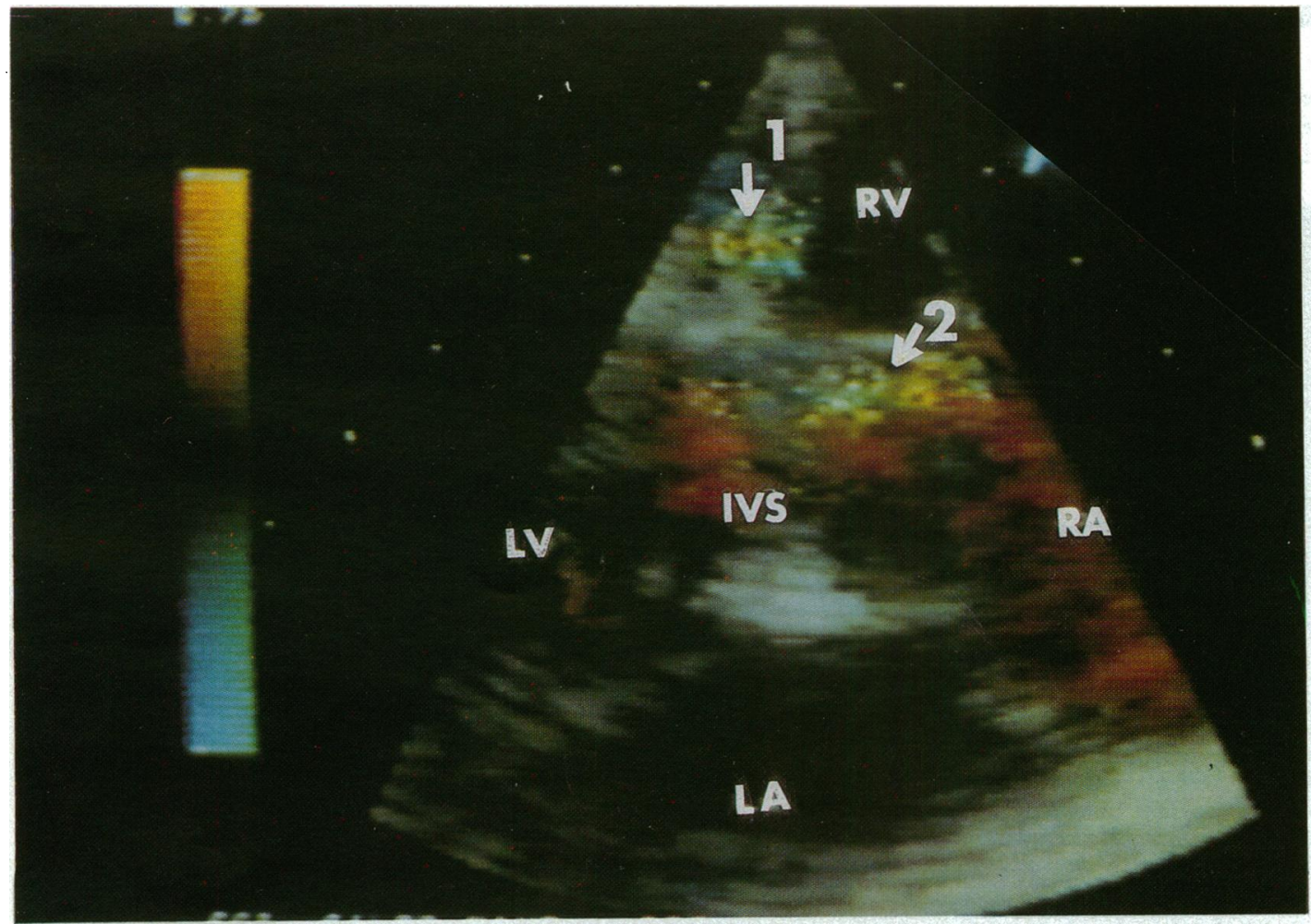

Fig 1 A modified four chamber view with the transducer angled towards the short axis view and recorded during systole in a patient with multiple restrictive ventricular septal defects. There are two mosaic jets exiting from the right ventricular aspect of the interventricular septum (arrows 1 and 2). In this view, the mosaic colour flow pattern completely traverses the septum only through the perimembranous defect (arrow 2). The midtrabecular defect (arrow 1) is depicted by a mosaic jet exiting from the right ventricular aspect of the septum. $L V$, left ventricle; $R V$, right ventricle; $L A$, left atrium; $R A$, right atrium; IVS, interventricular septum. 


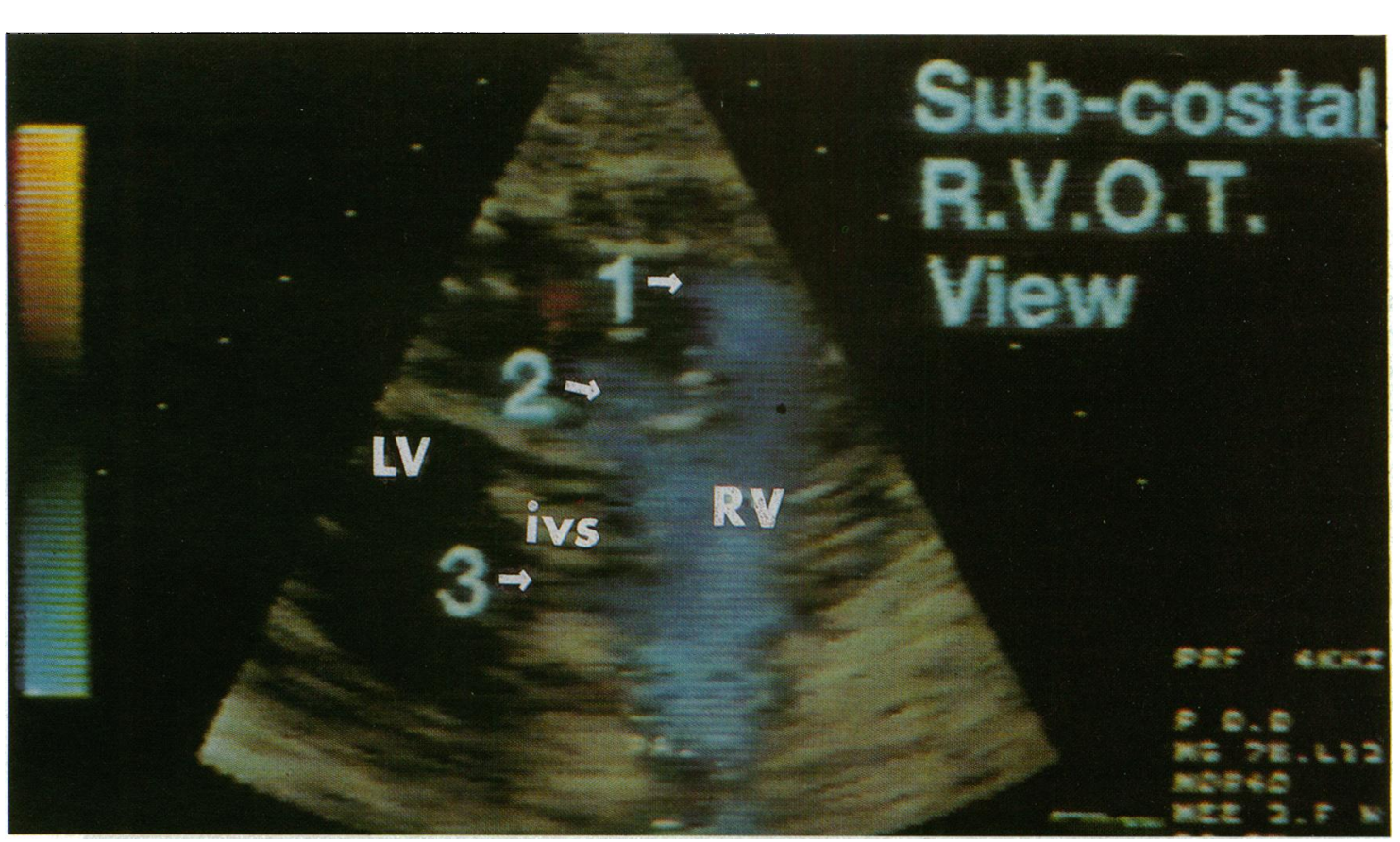

Fig 2 A subcostal view of the right ventricular outflow tract recorded during systole in a patient with multiple non-restrictive ventricular septal defects. Only low velocity laminar flow (blue) with no evidence of flow acceleration traverses the interventricular septum at the three points at which the ventricular septal defects were present (arrows 1,2, and 3). There is no aliasing or turbulence within the septum or the right ventricle. See legend to fig 1 for abbreviations.

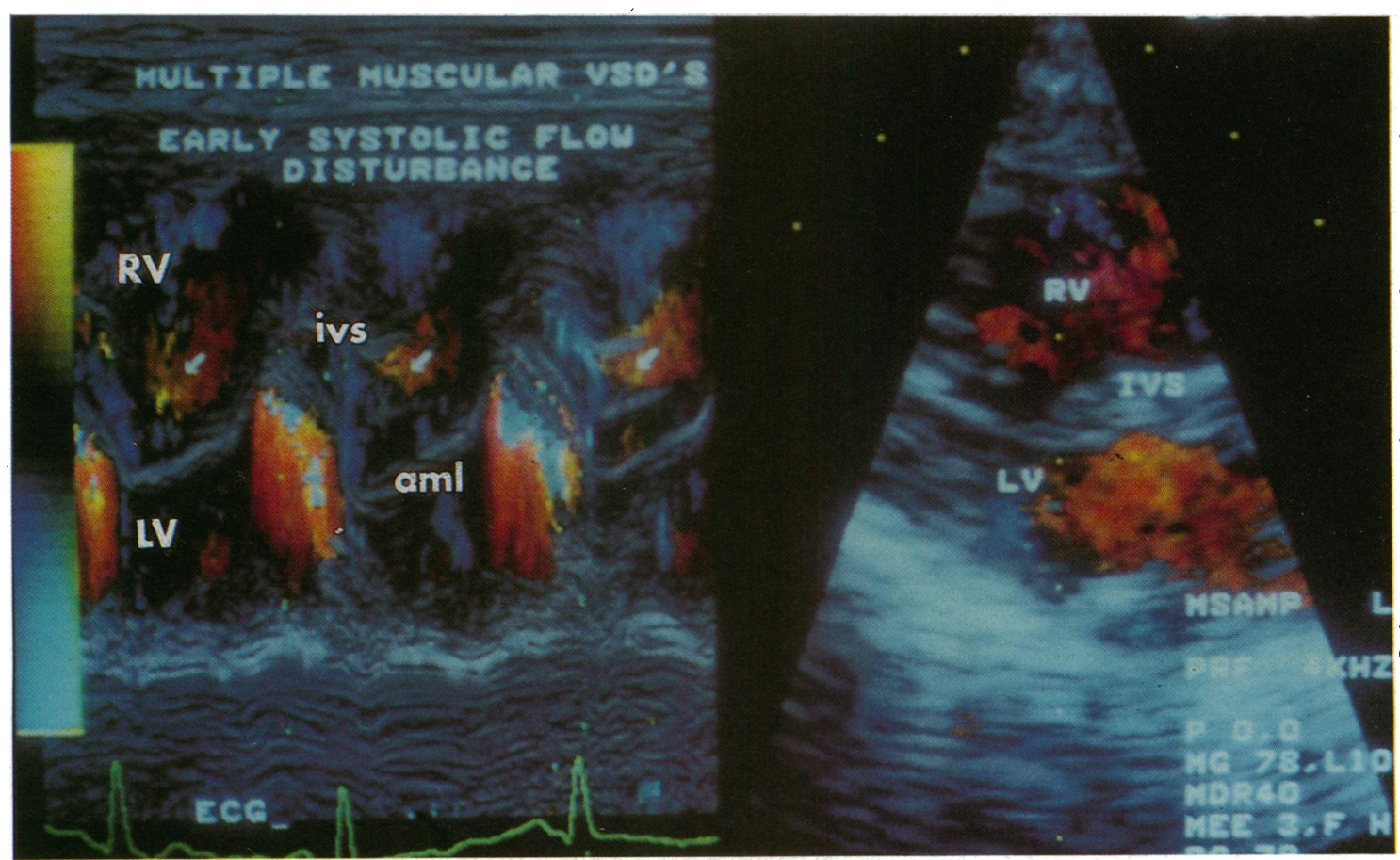

Fig $3 A$ colour flow map and colour $M$ mode recorded in the parasternal long axis view from a patient with non-restrictive multiple muscular ventricular septal defects and an appreciable left to right shunt. The colour flow map on the right is frozen in late systole; there is no turbulence within the septum or the right ventricle. In the colour $M$ mode on the left high velocity turbulent diastolic flow entering the left ventricular inflow was caused by high transmitral flow. In early systole there is colour flow traversing the interventricular septum and entering the right ventricle (arrows). The transseptal colour flow shows a colour shift from red to yellow indicating flow acceleration without turbulence or aliasing. See text for further details. aml, anterior mitral valve leaflet. See legend to fig 1 for other abbreviations. 
than colour flow imaging alone and thus could be a useful adjunct in the definition of multiple ventricular septal defects by detecting multiple discrete areas of acceleration of transseptal or right ventricular flow.

All cardiac catheterisation studies were performed under general anaesthesia with pressure recordings being taken before angiography. Left ventriculography was performed in the appropriate angled axial projections as described by Soto et al. ${ }^{13}$ Right ventriculography was also performed in all patients except those with isolated ventricular septal defects.

Although both echocardiographic and angiographic studies were sometimes performed by the same cardiologist in the same patient, all results were later assessed by one or more experienced independent observers.

Because these patients entered into the study in various ways it was not always possible to perform simultaneous angiographic and ultrasound studies. But both types of study were always performed within 24 hours of each other.

The results obtained were initially analysed as a total patient group and then further analysed after being subdivided into groups with restrictive or nonrestrictive defects. Non-restrictive defects were defined as showing equal peak systolic pressures in both ventricles measured by cardiac catheterisation. Patients with restrictive multiple ventricular septal defects had a peak systolic pressure in the left ventricle that exceeded that in the right ventricle, except patients with transposition of the great arteries in whom the systemic ventricle was on the right.

\section{Results}

Cross sectional echocardiography showed at least one ventricular septal defect in all 31 patients and one or more further defects in $12(38 \%)$ patients; there was no difference in the detection rate of multiple defects between the groups with restrictive (39\%) and nonrestrictive defects $(38 \%)$ respectively (table 2$)$. The diagnosis of multiple ventricular septal defects based on cross sectional imaging was subsequently confirmed in all 12 patients by a combination of either colour flow imaging, ventriculography, or operation or all three. In this series the addition of conventional pulsed and continuous wave Doppler to the cross sectional imaging information did not increase the detection of multiple ventricular septal defects. The conventional Doppler methods were useful for distinguishing between patients with restrictive or nonrestrictive haemodynamic function. In contrast, colour flow imaging alone, by visualising multiple areas of transseptal flow detected the presence of two
Table 2 Diagnostic accuracy of non-invasive and invasive techniques

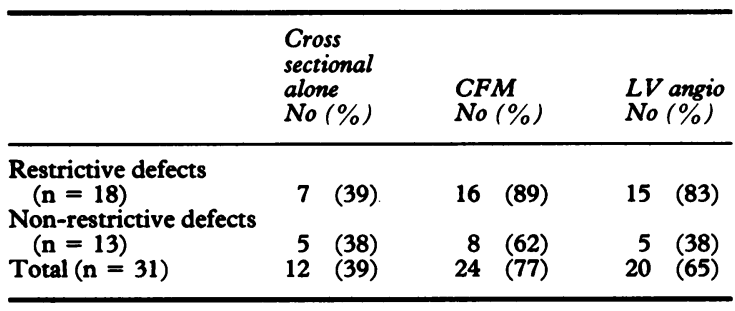

CFM, colour flow mapping; LV angio, left ventricular cineangiography.

or more ventricular septal defects in $24(77 \%)$ of the 31 patients studied. When these were subdivided into groups with restrictive and non-restrictive defects, colour flow imaging had a much higher diagnostic accuracy in the restrictive group (89\%) than in the non-restrictive groups $(62 \%)$. The diagnosis was confirmed in all 24 patients by either ventriculography or operation or both. A comparison of the different colour flow patterns obtained showed that there was no difficulty in distinguishing between restrictive (turbulent mosaic pattern) and non-restrictive (non-turbulent laminar flow pattern) defects. In patients with equal peak systolic ventricular pressures the detection of multiple ventricular septal defects depended on the ability to visualise colour encoded transseptal flow. The predominant direction of any shunt did not affect this ability but simply altered the colour of the transseptal flow (flow towards the transducer was encoded as red and flow away was encoded as blue).

Colour M mode studies showed transseptal flow in the largest defect in patients with non-restrictive ventricular septal defects, but could not confirm the diagnosis of multiple defects in any case. Transseptal flow was usually laminar but in one patient systolic flow acceleration was detected. This systolic flow acceleration was indicated by a change in colour intensity while flow itself remained laminar (fig 3). This pattern of flow acceleration correctly predicted the presence of equal systolic ventricular pressures and a significant left to right shunt (confirmed at catheterisation). All other patients with multiple non-restrictive defects had bidirectional shunting.

Left ventriculography showed multiple ventricular septal defects in only $20(65 \%)$ of the 31 patients. The diagnostic accuracy of ventriculography was $83 \%$ for restrictive defects and $38 \%$ for non-restrictive defects.

\section{Discussion}

The results of this study confirm that colour flow 
imaging is a useful adjunct in the non-invasive diagnosis of multiple ventricular septal defects. It proved to be better than cross sectional imaging alone, and we found that the addition of the conventional Doppler methods did not increase the diagnostic yield. Our results for the overall diagnostic accuracy of cross sectional imaging alone $(39 \%)$ and colour flow imaging $(77 \%)$ for multiple ventricular septal defects are similar to those previously reported by Ludomirsky and coworkers who showed an overall diagnostic sensitivity for cross sectional imaging and colour flow imaging of $38 \%$ and $72 \%$ respectively. ${ }^{11}$ When we subdivided our patients into groups with restrictive and non-restrictive defects, however, colour flow imaging was found to have a higher sensitivity for the diagnosis of multiple restrictive defects $(89 \%)$ ) than for multiple non-restrictive defects $(62 \%)$. This lower sensitivity for nonrestrictive defects seems to be a function of the absence of turbulent transseptal flow, which is a consequence of the equal peak systolic ventricular pressures. This was the fundamental difference between restrictive and non-restrictive defects as shown by colour flow imaging - turbulent high velocity flow encoded as a mosaic pattern was easier to visualise than low velocity laminar flow.

Diagnosis in the non-restrictive group was more difficult when a large ventricular septal defect (usually perimembranous) was found in combination with one or more small muscular defects; our study showed that under these circumstances colour flow information may only be visualised within the largest defect with no colour flow appearing in the small defect(s). This phenomenon was seen with both the velocity and power display modes. Whether this represented a true lack of flow in the smaller defects or was an artefact induced by either the analysis algorithm of the equipment or a failure to align correctly with defect flow is not clear, but if this is a true finding and flow can occur only over one of the multiple defects present, then it would in part explain the relatively high incidence of false negative diagnoses when colour flow imaging is used to identify multiple non-restrictive defects. The inherent higher sampling rate of colour $M$ mode might in theory detect transseptal flow in these small additional defects that were missed by colour flow mapping. In practical terms, however, this was not the case because colour $M$ mode only detected transseptal flow in the largest defect present.

The combination of isolated small multiple ventricular septal defects and pulmonary stenosis (or pulmonary band) might make diagnosis difficult, although this particular anatomical arrangement was not encountered in this series. Such an arrangement causes non-restrictive haemodynamic function and equal peak systolic ventricular pressures, but if the pulmonary obstruction were to be removed then the defects would be restrictive (because they are small) and might therefore not require concomitant closure. The data described above suggest that such multiple small defects will not be reliably visualised by colour flow imaging.

There were other difficulties with colour flow imaging, both in the groups with restrictive and nonrestrictive defects when multiple ventricular septal defects lay close together. The limitation of lateral resolution inherent in the technique do not allow the separation of turbulent jets that lie within $<0.5 \mathrm{~cm}$ of each other. In contrast, the combination of restrictive perimembranous and apical trabecular defects was accurately defined because the turbulent jets were well separated and there was no difficulty with lateral resolution. Also the detection of multiple defects in the outlet muscular septum (either restrictive or non-restrictive) will always prove difficult both because of the difficulties in imaging this region and the subsequent difficulties with lateral resolution with colour flow imaging. We believe that these are the reasons for the diagnostic failures in this study.

The overall diagnostic accuracy of left ventriculography for multiple ventricular septal defects reported in this study $(65 \%)$ is lower than that reported by Fellows and coworkers $(86 \%)^{5}$. There are probably two reasons for this. Firstly, the proportion of restrictive defects in their study group was much higher-so the diagnostic accuracy of restrictive defects in our study $(83 \%)$ is a more appropriate comparison and does accord with their findings. Secondly, their study was not primarily designed to assess the diagnostic accuracy of their technique and it is possible that some cases that were missed by left ventriculography were not confirmed by surgical inspection (that is where a palliative procedure such as a pulmonary artery band or a systemic-pulmonary shunt was performed rather than a total correction). This would be particularly applicable to patients with non-restrictive defects in whom the diagnosis by left ventriculography in this study was especially poor $(38 \%)$.

The relative diagnostic accuracy of colour flow imaging $(89 \%)$ and left ventriculography $(83 \%)$ for all types of multiple restrictive ventricular septal defects in our study was similar. There were no cases in this subgroup that were diagnosed by left ventriculography but not by colour flow imaging. So left ventriculography was of no additional benefit in patients in this study with multiple restrictive defects. In patients with multiple non-restrictive defects, the results were different; in this subgroup there were two patients in whom the diagnosis was made by left ventriculography but was missed by 
colour flow imaging. So although the diagnostic accuracy for colour flow imaging (65\%) was much higher than that of left ventriculography (38\%), the overall diagnostic accuracy for multiple non-restrictive ventricular septal defects was highest when the information from the two techniques was used together $(77 \%)$.

None the less, $23 \%$ of multiple non-restrictive defects were misdiagnosed as single isolated ventricular septal defects. We feel that this is a fair reflection on the complexity of the diagnosis presented by such defects. It is our opinion that the diagnostic yield will be only slightly increased with more experience in the use of the diagnostic techniques described above and that up to one in five of multiple non-restrictive defects and one in 10 of multiple restrictive defects may be missed.

We conclude that colour flow imaging was the single most accurate diagnostic technique for the prediction of multiple restrictive ventricular septal defects. Whereas the diagnosis of multiple non-restrictive defects was best achieved in this study when colour flow imaging and left ventriculography were used together, even the combined approach missed a considerable number of patients with multiple ventricular septal defects.

\section{References}

1 Friedman WF, Mehrizi A, Pusch AL. Multiple muscular ventricular septal defects. Circulation 1965; 32:35-42.

2 Fox KM, Patel RG, Graham GR, et al. Multiple and single ventricular septal defect. A clinical and haemodynamic comparison. Br Heart J 1978;40: 141-6.

3 Breckenbridge IM, Stark J, Waterson DJ, Bonham-
Carter RE. Multiple ventricular septal defects. Ann Thorac Surg 1972;13:128-36.

4 Wilkinson JL, Wilcox BN, Anderson RH. The anatomy of double outlet right ventricle. In: Anderson $\mathbf{R H}$, Macartney FJ, Shinebourne EA, Tynan M, eds. Paediatric cardiology Vol 5. Edinburgh: Churchill Livingstone, 1981:397-407.

5 Fellows KE, Westerman MD, Keane JF. Angiography of multiple ventricular septal defects in infancy. Circulation 1982;66:1094-9.

6 Soto B, Bargeron LM, Pacifico AD, Vanini V, Kirklin JW. Angiography of atrioventricular canal defects. Am J Cardiol 1981;48:492-9.

7 Kirklin JK, Castaneda AR, Keane JF, Fellows KE, Norwood WI. Surgical management of multiple ventricular septal defects. $J$ Thorac Cardiovasc Surg 1980;80:485-93.

8 Taylor JFN, Chrispin AR. Interventricular septal defect shown by left ventricular cine-angiocardiography. Br Heart J 1971;33:285-9.

9 Sutherland GR, Godman MJ, Smallhorn JF, Guiterras $P$, Anderson RH, Hunter S. Ventricular septal defects. Two dimensional echocardiographic and morphological correlations. Br Heart J 1982;47: 316-28.

10 Magherini A, Simonetti L, Tomassini CR, Moggi C, Ragazzini F, Bartolozzi G. Cross-sectional echocardiography with pulsed and continuous wave Doppler in the management of ventricular septal defects. Int $J$ Cardiol 1987;15:317-28.

11 Ludomirsky A, Huhta JC, Vick GW, Murphy DJ, Danford DA, Morrow WR. Color Doppler detection of multiple ventricular septal defects. Circulation 1986;74:1317-22.

12 Ortiz E, Robinson PJ, Deanfield JE, Franklin R, Macartney FJ, Wyse RKH. Localisation of ventricular septal defects by simultaneous display of superimposed colour Doppler and cross sectional echocardiographic images. Br Heart J 1985;54:53-60.

13 Soto B, Coghlan CH, Bargeron LM Jr. Angiography in ventricular septal defects. In: Anderson RH, Shinebourne EA, eds. Paediatric cardiology. Edinburgh: Churchill Livingstone, 1978:125-35. 